\title{
Código Infarto en una unidad de cuidados intermedios cardiológicos en época de COVID-19
}

\author{
Infarction code in a cardiac intermediate care unit during COVID-19 pandemic \\ Marcos Farráis-Villalba, Javier Lorenzo-González, Nerea Torres-González*, María Facenda-Lorenzo, \\ María Ramos-López y Julio S. Hernández-Afonso \\ Servicio de Cardiología, Hospital Universitario Nuestra Señora de Candelaria, Santa Cruz de Tenerife, España
}

\section{Sr. Editor:}

Mientras nos enfrentamos a la incógnita de si el coronavirus SARS-CoV-2 podrá erradicarse o se convertirá en un virus estacional, asistimos al temido escenario de las sucesivas olas epidémicas que ponen en dificultades a los servicios de salud, todavía muy afectados tras el primer azote de la pandemia. La ocupación hospitalaria por pacientes con COVID ha obligado a realizar cambios organizativos, suspender la actividad asistencial no urgente e incrementar el número de camas. Como parte del plan de contingencia por la pandemia por COVID-19 de nuestro hospital, un centro de tercer nivel con un área de 500,000 habitantes y una capacidad de 900 camas de hospitalización, y con el fin de aliviar la presión asistencial sobre la unidad de medicina intensiva, se decidió ingresar a los pacientes del programa Código Infarto en la unidad de cuidados intermedios cardiológicos (UCIC), que cuenta con 16 camas y capacidad para vigilancia electrocardiográfica continua, con un monitor situado en el control de enfermería que permite la visualización de las telemetrías y el control de las alarmas. No fue preciso llevar a cabo ninguna adecuación de la unidad, ya que se encuentra en funcionamiento desde el 2014; sin embargo, hasta el inicio de la pandemia sólo ingresaban pacientes de nivel I de complejidad, según el documento de posicionamiento sobre las unidades de cuidados intensivos cardiológicos de la Asociación de Cuidados
Cardiovasculares Agudos de la Sociedad Europea de Cardiología ${ }^{1}$. Dada la situación de sobrecarga asistencial y el gran número de profesionales sanitarios que se encontraban de baja médica por infección o contacto estrecho con pacientes con COVID, no se consideró disminuir el cociente enfermera/paciente, de tal forma que en cada turno había 1 enfermero por cada 4 camas de la unidad de cuidados intermedios y 6 camas de hospitalización convencional. En 2007, la Sección de Cardiopatía isquémica y Unidades Coronarias de la Sociedad Española de Cardiología ${ }^{2}$ describió las indicaciones de ingreso en una UCIC; una de ellas es la del síndrome coronario agudo con elevación del segmento ST (SCACEST no complicado tras realizar la revascularización). En nuestro caso, se trataba de pacientes con SCACEST sometidos a angioplastia primaria emergente, así como aquellos en los que se llevaba a cabo fibrinólisis por encontrarse en regiones periféricas en las que el tiempo desde el primer contacto médico hasta su llegada a nuestro centro era superior a 90 minutos. La seguridad de esta medida ya la han valorado otros grupos ${ }^{3}$ y la propia Sociedad Española de Cardiología insta a los servicios de cardiología a adaptarse para participar en el tratamiento del paciente agudo cardiológico ${ }^{4}$.

El protocolo de actuación se describe en la figura 1. Entre el 11 de marzo y el 25 de septiembre de 2020 ingresaron en la UCIC 112 pacientes (77.7\% varones
Correspondencia:

*Nerea Torres-González

E-mail: nereatorresg@gmail.com
Disponible en internet: 14-07-2021 Arch Cardiol Mex. 2021;91(Supl):134-137 www.archivoscardiologia.com 1405-9940 / @ 2021 Instituto Nacional de Cardiología Ignacio Chávez. Publicado por Permanyer. Este es un artículo open access bajo la licencia CC BY-NC-ND (http://creativecommons.org/licenses/by-nc-nd/4.0/). 


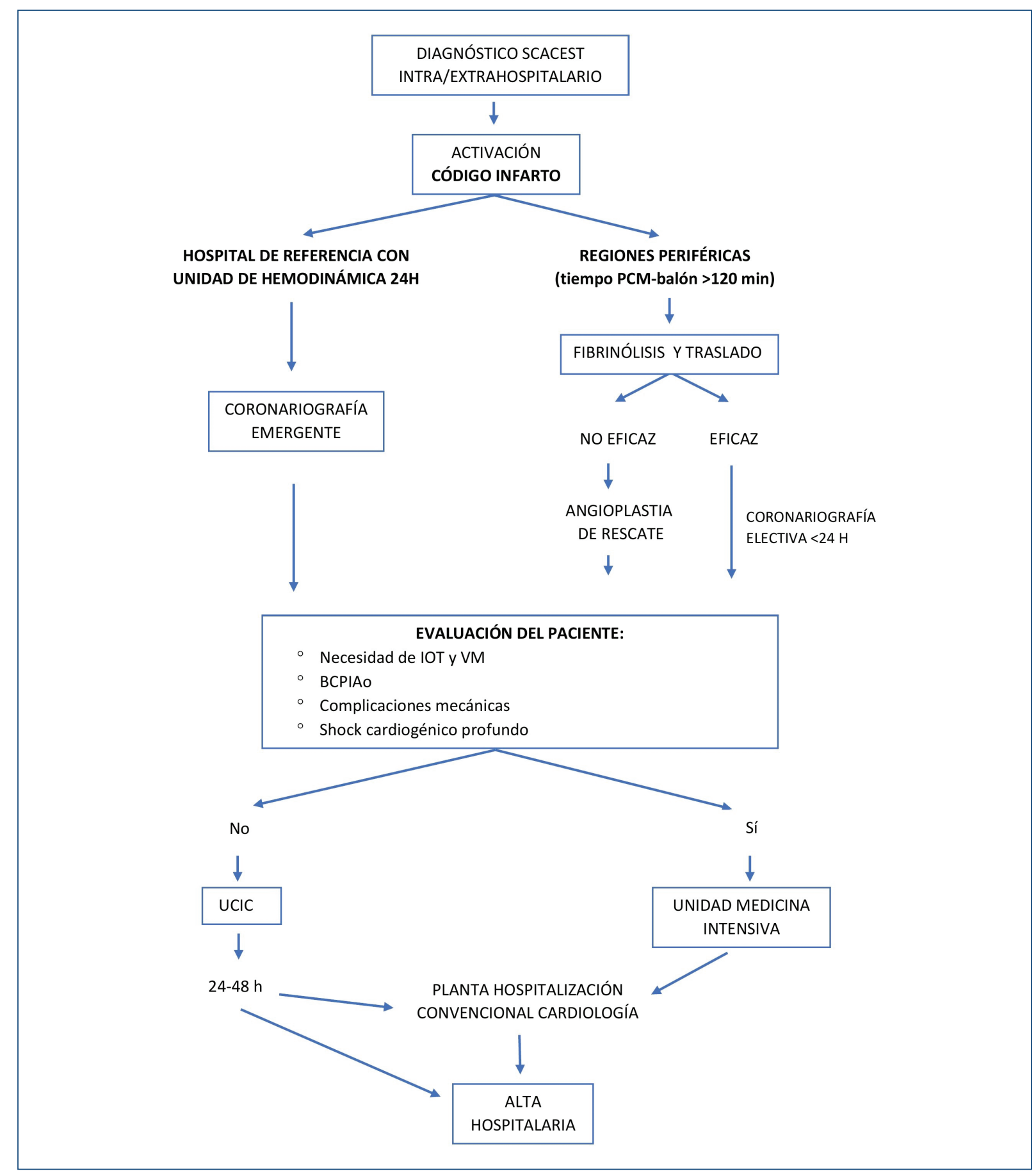

Figura 1. Protocolo de actuación y diagrama de decisiones en el ingreso del SCACEST. Tiempo PCM-balón: tiempo desde el primer contacto médico hasta la insuflación del balón durante la angioplastia; IOT: intubación orotraqueal; VM: ventilación mecánica; BCPIAo: balón de contrapulsación intraaórtico; UCIC: unidad de cuidados intermedios cardiológicos.

con edad de $58.6 \pm 12.4$ años). En la tabla 1 se expone un resumen de los resultados. La presentación del infarto según la clasificación de Killip y Kimball fue la siguiente: Killip I en $87.5 \%$, seguida por Killip II en $8.9 \%$ y sólo 4 pacientes se presentaron como Killip III y IV. En el $97.3 \%$ se realizó angioplastia primaria y en 3 enfermos (2.7\%) se efectuó fibrinólisis, que fue eficaz por criterios clínicos y electrocardiográficos en dos de ellos, no así en un tercero que requirió angioplastia de rescate. Con respecto a la arteria causante del 
Tabla 1. Principales factores de riesgo cardiovascular y resumen de los resultados

\begin{tabular}{|c|c|c|}
\hline & $\begin{array}{l}\text { Pacientes } \\
\text { (n) }\end{array}$ & Porcentaje \\
\hline \multicolumn{3}{|l|}{ Factores de riesgo } \\
\hline Tabaquismo & 60 & 53.6 \\
\hline Extabaquismo & 21 & 18.8 \\
\hline Hipertensión arterial & 58 & 51.8 \\
\hline Dislipemia & 56 & 50 \\
\hline $\begin{array}{l}\text { Diabetes mellitus } \\
\text { Tipo } 1 \\
\text { Tipo } 2\end{array}$ & $\begin{array}{c}1 \\
27\end{array}$ & $\begin{array}{c}0.9 \\
24.1\end{array}$ \\
\hline Cardiopatía isquémica previa & 10 & 8.9 \\
\hline \multirow{2}{*}{$\begin{array}{l}\text { Enfermedad renal crónica (filtrado } \\
\text { glomerular }<60 \mathrm{ml} / \mathrm{kg} / 1.73 \mathrm{~m}^{2} \text { ) }\end{array}$} & 8 & 7.2 \\
\hline & $\begin{array}{l}\text { Pacientes } \\
\text { (n) }\end{array}$ & Porcentaje \\
\hline \multicolumn{3}{|l|}{ Características de los pacientes } \\
\hline Género masculino & 87 & 77.7 \\
\hline $\begin{array}{l}\text { Clasificación de Killip y Kimball } \\
\text { Killip I } \\
\text { Killip II } \\
\text { Killip III } \\
\text { Killip IV }\end{array}$ & $\begin{array}{c}98 \\
10 \\
2 \\
2\end{array}$ & $\begin{array}{l}87.5 \\
8.9 \\
1.8 \\
1.8\end{array}$ \\
\hline Angioplastia primaria emergente & 109 & 97.3 \\
\hline Fibrinólisis & 3 & 2.7 \\
\hline $\begin{array}{l}\text { Arteria causante del SCACEST } \\
\text { Coronaria derecha } \\
\text { Descendente anterior } \\
\text { Circunfleja } \\
\text { Vaso secundario no } \\
\text { revascularizable }\end{array}$ & $\begin{array}{c}42 \\
42 \\
25 \\
3\end{array}$ & $\begin{array}{c}37.5 \\
37.5 \\
22.3 \\
2.7\end{array}$ \\
\hline Necesidad de VMNI & 2 & 1.8 \\
\hline Necesidad de aminas vasoactivas & 4 & 3.6 \\
\hline $\begin{array}{l}\text { Necesidad de marcapasos } \\
\text { transitorio }\end{array}$ & 8 & 7.1 \\
\hline
\end{tabular}

síndrome coronario, las más frecuentes fueron la coronaria derecha y la descendente anterior con una frecuencia del $37.5 \%$ en ambas, seguida de la arteria circunfleja en el $22.3 \%$ de los pacientes y en el $2.7 \%$ se trataba de un vaso secundario. Durante el ingreso, 2 pacientes $(1.8 \%)$ requirieron ventilación mecánica no invasiva, 4 (3.6\%) precisaron apoyo inotrópico con aminas vasoactivas y fue necesario el implante de marcapasos transitorio en 8 (7.1\%) por bloqueo auriculoventricular de alto grado transitorio. En cuanto al grado de complejidad, la mayoría de los pacientes era de nivel II (97.3\%) y 3 pacientes pertenecían al nivel III, dos por paro cardiorrespiratorio recuperado y uno por choque cardiogénico. Estos pacientes de nivel III de complejidad mejoraron su situación hemodinámica y respiratoria tras la angioplastia, por lo que pudieron ingresar en la unidad de cuidados intermedios cardiológicos. Ningún paciente requirió programarse para operación cardíaca; sin embargo, dos pacientes que presentaron al principio un SCACEST en situación Killip I se trasladaron con posterioridad a la unidad de medicina intensiva, ambos por complicación en forma de trombosis aguda del stent. Uno de ellos padecía una discrasia sanguínea que lo predisponía a la hipercoagulabilidad y sufrió fibrilación ventricular (FV) que se desfibriló con rapidez sin deterioro respiratorio; este individuo regresó a la UCIC luego de la angioplastia para tratamiento de la trombosis del stent, bajo perfusión de anti-Ilb/llla, pese a lo que presentó un nuevo episodio de FV por nueva trombosis del stent y se decidió su traslado a la unidad de cuidados intensivos. La segunda paciente mostró una FV que derivó rápidamente en asistolia, por lo que requirió intubación orotraqueal y ventilación mecánica antes del traslado a la sala de hemodinámica. La estancia media hospitalaria fue de $3.73 \pm 1.9$ días; debe considerarse que la revascularización de los pacientes que tenían lesiones graves distintas de las que causó el SCACEST se realizó en un segundo tiempo durante el ingreso hospitalario. La fracción de expulsión del ventrículo izquierdo (FEVI) media al alta fue de $50.5 \pm 10.4 \%$. Durante el período de seguimiento intrahospitalario se registró un solo fallecimiento, un varón de 87 años con SCACEST inferior sometido a angioplastia primaria sobre la circunfleja, con una FEVI del $45 \%$ y sin complicaciones cardiológicas (se realizó una flebitis sobre una vía periférica, se lo trasladó a la planta de hospitalización de cardiología y allí sufrió una muerte súbita). El 3.6\% (4 pacientes) requirió reingreso antes del mes desde el alta, dos por insuficiencia cardíaca congestiva, uno con lesiones coronarias significativas no revascularizables que reingresó por un síndrome coronario agudo sin elevación del ST, y otro más que ingresó por choque cardiogénico resistente y que representó la única muerte en el período del primer mes tras el ingreso por SCACEST.

En las últimas décadas hemos asistido a un cambio en el paradigma terapéutico de los pacientes con SCACEST, tanto por la universalización de la angioplastia primaria y la implementación del sistema de "código infarto" que ha mejorado sustancialmente los tiempos de actuación, como por la creación de las unidades 
coronarias y las unidades de cuidados intermedios cardiológicos. Existe controversia acerca de los resultados de mortalidad en estos pacientes según sea que se los trate en unidades de cuidados intensivos o en unidades coronarias asistidas por cardiólogos ${ }^{5-9}$, datos difícilmente comparables debido a diseños dispares en los estudios, realizados en países y etapas diferentes, y probablemente con criterios distintos para establecer qué pacientes pueden beneficiarse de ingresar a una $u$ otra unidad. Las guías europeas y estadounidense del síndrome coronario agudo con elevación del ST destacan la necesidad de la vigilancia estrecha y la supervisión de los pacientes después del procedimiento de la angioplastia debido al alto riesgo arrítmico, si bien esta vigilancia puede proporcionarse en ambas unidades. Existe abundante bibliografía que analiza el hecho de que existe un perfil de paciente con SCACEST y reperfusión temprana que tiene bajo riesgo de complicaciones y podría tratarse en una unidad de cuidados intermedios, con la subsecuente liberación de camas en cuidados intensivos y ahorro en gasto sanitario ${ }^{10-12}$.

En nuestra experiencia, el ingreso en una unidad de cuidados intermedios cardiológicos de pacientes con SCACEST no complicado tratado con intervencionismo coronario percutáneo temprano es una conducta segura y tiene una tasa muy baja de complicaciones. A pesar de las limitaciones de este estudio, por ejemplo su carácter retrospectivo y el tamaño insuficiente de la muestra, según nuestros resultados, esta estrategia no empeora los datos de mortalidad en estos pacientes, se facilita la liberación de camas en la unidad de medicina intensiva, de vital importancia en la crisis sanitaria actual, y probablemente contribuye a disminuir la estancia media hospitalaria y los costos sanitarios.

\section{Financiamiento}

Los autores declaran no haber recibido financiación para este estudio.

\section{Conflicto de intereses}

Los autores declaran no tener conflicto de intereses.

\section{Responsabilidades éticas}

Protección de personas y animales. Los autores declaran que para esta investigación no se han realizado experimentos en seres humanos ni en animales.

Confidencialidad de los datos. Los autores declaran que han seguido los protocolos de su centro de trabajo sobre la publicación de datos de pacientes.

Derecho a la privacidad y consentimiento informado. Los autores declaran que en este artículo no aparecen datos de pacientes.

\section{Bibliografía}

1. Bonnefoy-Cudraz E, Bueno H, Casella G. Editor's choice - acute cardiovascular care association position paper on intensive cardiovascular care units: an update on their definition, structure, organisation and function. European Heart Journal: Acute Cardiovascular Care. 2018;7(1):80-95. doi:10.1177/2048872617724269

2. Alonso JJ, Sanz G, Guindo J, García-Moll X, Bardají A, Bueno H. Unidades de cuidados intermedios: base racional, infraestructura, equipamiento e indicaciones de ingreso. Rev Esp Cardiol.2007;60:404-14

3. Viana-Tejedor A, López De Sá E, Peña-Conde L. Do patients with ST segment elevation myocardial infarction in Killip class I need intensive cardiac care after a successful primary percutaneous intervention? Acute Card Care. 2009;11(4):243-6. doi: 10.1080/17482940903362588.

4. Worner F, Román A, Sánchez PL, Tejedor A, González-Juanatey J. Atención a los pacientes con enfermedades cardíacas agudas y críticas. Posición de la Sociedad Española de Cardiología. Rev Esp Cardiol. 2016;69:239-242.

5. Miki R, Takeuchi M, Imai T, Seki T, Tanaka S, Nakamura M, et al. Association of intensive care unit admission and mortality in patients with acute myocardial infarction. Journal of Cardiology. 2019;74:10.1016/j. jjcc.2019.01.007.

6. Valley TS, Iwashyna TJ, Cooke CR, Sinha SS, Ryan AM, Yeh RW, et al. Intensive care use and mortality among patients with ST elevation myocardial infarction: retrospective cohort study. BMJ 2019;365:11927 doi:10.1136/bmj.l1927

7. D'Souza M, Saaby L, Poulsen T, Diederichsen A, Hosbond S, Diederichsen SZ, et al. Comparison of mortality in patients with acute myocardial infarction accidentally admitted to non-cardiology departments versus that in patients admitted to coronary care units. The American Journal of Cardiology. 2014;114:10.1016/j.amjcard.2014.07.035.

8. Fanaroff A, Peterson E, Chen A, Thomas L, Doll J, Fordyce C, et al. Intensive Care unit utilization and mortality among Medicare patients hospitalized with non-ST-segment elevation myocardial infarction. JAMA Cardiology. 2016;2:10.1001/jamacardio.2016.3855.

9. Bosch X, Pérez J, Ferrer E, Ortiz-Pérez J, Pérez-Alba J, Heras M, et al. Perfil clínico, tratamiento y pronóstico de los pacientes con infarto agudo de miocardio no ingresados en una unidad coronaria: utilidad de una unidad de cuidados intermedios como lugar de ingreso inicial. Rev Esp Cardiol. 2003;56:262-270. 10.1157/13043952.

10. Shavadia J, Chen A, Fanaroff A, Lemos J, Kontos M, Wang J. Intensive care utilization in stable patients with ST-segment elevation myocardial infarction treated with rapid reperfusion. JACC: Cardiovascular Interventions. 2019;12:709-717. 10.1016/j.jcin.2019.01.230.

11. Norton JM, Reddy PK, Subedi K, Fabrizio CA, Wimmer NJ, Urrutia LE. Utilization of an ICU severity of illness scoring system to triage patients with ST-elevation myocardial infarction. J Intensive Care Med. 2020;10:885066620928263. doi: 10.1177/0885066620928263. Epub ahead of print. PMID: 32519573.

12. Chen R, Strait KM, Dharmarajan K. Hospital variation in admission to intensive care units for patients with acute myocardial infarction. Am Heart J. 2015;170(6):1161-1169. doi:10.1016/j.ahj.2015.09.003 